\title{
miR-326 inhibits the cell proliferation and cancer stem cell-like property of cervical cancer in vitro and oncogenesis in vivo via targeting TCF4
}

\author{
Jian Zhang ${ }^{1 \#}$, Haining He ${ }^{1 \#}$, Kana Wang ${ }^{2}$, Yao Xie ${ }^{1}$, Zhongmei Yang ${ }^{1}$, Mingrong Qie ${ }^{2}$, Zhi Liao ${ }^{1}$, \\ Zhenrong Zheng'
}

${ }^{1}$ Department of Obstetrics and Gynecology, Sichuan Academy of Medical Sciences \& Sichuan Provincial People's Hospital, Chengdu, China; ${ }^{2}$ Department of Obstetrics and Gynecology, West China Second University Hospital, Sichuan University, Chengdu, China

Contributions: (I) Conception and design: J Zhang, H He; (II) Administrative support: Z Liao; (III) Provision of study materials or patients: All authors; (IV) Collection and assembly of data: All authors; (V) Data analysis and interpretation: All authors; (VI) Manuscript writing: All authors; (VII)

Final approval of manuscript: All authors.

"These authors contributed equally to this work.

Correspondence to: Zhi Liao; Zhenrong Zheng. No. 32, West Second Section, First Ring Road, Chengdu 610072, China.

Email: Liaozhi130@163.com; 675318179@qq.com.

Background: Cervical cancer ranks as one of the most prevalent female malignancies globally, and its treatment with new targets has been the focus of current research. The present study set out to investigate the function of microRNA-326 (miR-326) in vitro and in vivo and to verify the direct targeting of transcription factor 4 (TCF4) by miR-326.

Methods: The detection of messenger RNA (mRNA) expressing miR-326 and TCF4 in cervical cancer cell lines and tumor samples was conducted using quantitative real-time polymerase chain (qRT-PCR). A dualluciferase reporter assay was carried out to detect the target relationship of miR-326 with TCF4. A Cell Counting Kit-8 (CCK-8) assay was employed to detect the effect of miR-326 on CasKi cell viability. Flow cytometry and western blotting were employed to examine the effects of miR-326 on cancer stem cell (CSC)like property. Tumor weight was measured in orthotopic xenograft mouse models. Immunohistochemistry was employed to analyze the protein expression levels of Ki-67, proliferating cell nuclear antigen (PCNA), CD44, and SRY-box 4 (SOX4).

Result: Downregulation of the mRNA expression levels of miR-326 was observed in cervical cancer cell lines and tumor tissue, while the levels of TCF4 were upregulated. The dual-luciferase reporter assay revealed binding of miR-326 to the three prime untranslated region (3'-UTR) of TCF4. In vitro assays demonstrated that miR-326 inhibited CasKi cell proliferation through regulating TCF4. miR-326 also suppressed the CSC-like property of CasKi cells by targeting TCF4. Furthermore, the protein expression levels of cyclin D1, $\beta$-catenin, and c-Myc were decreased when miR-326 was added to TCF4-transfected cells. In vivo assays demonstrated that miR-326 inhibited tumor weight, growth, and the protein expression levels of Ki-67, PCNA, CD44, SOX4, and $\beta$-catenin.

Conclusions: miR-326 acted in a tumor-suppressive manner through its regulation of TCF4, and has potential as a biomarker or therapeutic target for cervical cancer.

Keywords: microRNA-326 (miR-326); cell proliferation; cancer stem cell-like property (CSC-like property); CasKi cell; transcription factor 4 (TCF4)

Submitted Sep 03, 2020. Accepted for publication Dec 17, 2020.

doi: $10.21037 / \mathrm{atm}-20-6830$

View this article at: http://dx.doi.org/10.21037/atm-20-6830 


\section{Introduction}

Cervical cancer (CC) ranks third for incidence among all female malignancies globally and is responsible for the deaths of $\sim 20$ million women annually. A $50-75 \%$ decrease in CC mortality has occurred over the past 50 years, but the disease remains the third most common cancer in developing countries (1). Squamous cell carcinoma (SCC) and adenocarcinoma (AC) constitute the main histologic types of cervical cancer. Among total CC cases, 85-90\% are SCC. Persistent infection with high-risk human papillomavirus (HPV) is the principal causative factor of CC (2,3). SiHa (SCC, HPV16), CaSki (metastasized SCC, HPV16), and HeLa (AC, HPV18) are all CC cell lines (4). Epigenetic alteration due to abnormalities in DNA methylation and histone modification in CC has been the focus of extensive study. Recently, research strategies have provided a comprehensive insight on non-coding RNAs, particularly microRNAs (miRNAs) and long noncoding RNA (lncRNAs) (5). Furthermore, miRNAs have been identified as important regulators of proliferation, migration, invasion, and apoptosis in tumor cells (6). In short, miRNAs serve a critical role in CC progression (7). Other research has proposed that microRNA-326 (miR326) makes a contribution to a variety of malignancies, including colorectal cancer (8), hepatocellular carcinoma (9), breast cancer (10), and endometrial carcinoma (11). Importantly, downregulation of miR-326 has been reported in CC tissues and cells (12). In addition, the downregulation of lncRNA testis developmental-related gene 1 (TDRG1) has been found to enhance the expression levels of miR326 and in turn decrease mitogen-activated protein kinase 1 (MAPK1) levels, thereby alleviating CC cell proliferation and invasion (13). Altogether, these findings suggest that miR-326 might play a critical part in CC.

Transcription factor 4 (TCF4) is a pivotal transcription factor that interacts with $\beta$-catenin to trigger target gene transcription in response to Wnt activation (14). TCF4 plays a carcinogenic role in epithelial ovarian cancer, and TCF4 is also a prognostic biomarker for epithelial ovarian cancer (15). Crucially, research has verified that restoring TCF4 has an attenuation effect on the tumorsuppressive activity of miR-139-5p in CC progression and can oversee normal $\mathrm{Wnt} / \beta$-catenin signaling recovery (16). Interestingly, the relevant evidence indicates that miR-1184, miR-326, and miR-330-5p are involved in colorectal cancer via interacting with TCF4, C16orf45, and Ras Guanine-Releasing Protein 3 (RasGRP3) (17).
Furthermore, in the search for the potential targets of miR326 , bioinformatics algorithms have revealed that miR326 contains complementary elements to the three prime untranslated region (3'-UTR) of TCF4 regions. Taking into account these results, we hypothesized that miR-326 might be critical to regulating TCF4 expression and could thus be used to suppress the progression of CC.

Cancer stem cells (CSCs) are understood to be a small tumor cell subpopulation with involvement in tumorigenesis, multilineage differentiation potential, selfrenewal, slow cycling capacity, and tumorigenicity (18). CC stem cells (CCSCs) are heterogenous in cervical carcinoma, and cervical carcinoma tissue has been found to partly consist of diversely differentiated carcinoma cells (19). In recent research, CSCs were also shown to possess the capability of transdifferentiation into vascular endothelial cells and other tumor-associated stromal cells (20), which may also give rise to tumor heterogeneity. Research has proven that miR-326 inhibits the activation of the G-protein-coupled receptor 91 (GPR91)/signal transducer and activator of transcription 3 (STAT3)/vascular endothelial growth factor (VEGF) signaling pathway and significantly attenuates the activity of stem cells in endometrial carcinoma, both in vitro and in vivo (21). Moreover, downregulation of miR-326 has been suggested to be the mechanism underlying the unrestricted activation of the smoothened (Smo) signal transducer of the oncogenic Hedgehog (Hh) pathway in chronic myeloid leukemia. In addition, the restoration of miR-326 expression could also contribute to eradicating $\mathrm{CD} 34^{+}$chronic myeloid leukemia stem/progenitor cells (22). CD133 may be utilized as a CCSC-specific marker in patients undergoing CSCtargeted therapy (23). Elevation of ALDH1 expression in CC cells is linked to high rates of cell proliferation, sphere formation, migration, and tumorigenesis, which suggests that it functions as a stemness factor in CC (24). Based on these results, we hypothesized that miR-326 might regulate the CSC-like property of CC.

In the current study, the effect of miR-326 on cell proliferation and CSC-like property of SCC CasKi cells in vitro was examined. Furthermore, the targeting relationship of miR-326 with TCF4 was analyzed by conducting a dual-luciferase reporter assay. From this, miR-326 was found to perform a tumor-suppressive function through its targeting of TCF4 in vitro and in vivo. The results of this study can serve as a foundation for the future utilization of miR-326 in tumor suppression in CC therapy. 
We present the following article in accordance with the ARRIVE reporting checklist (available at http://dx.doi. org/10.21037/atm-20-6830).

\section{Methods}

\section{Cell culture}

The CC cell lines HeLa, CasKi, C4-1, and SiHa, along with the normal CC line Ect1/E6E7, were supplied by the American Type Culture Collection (ATCC, Manassas, VA, USA). Etc1/E6E7 cells were kept in keratinocyte serumfree medium (Gibco, Carlsbad, CA, USA) with $0.1 \mathrm{ng} / \mathrm{mL}$ human recombinant epidermal growth factor (EGF), $0.05 \mathrm{mg} / \mathrm{mL}$ bovine pituitary extract, and an additional $44.1 \mathrm{mg} / \mathrm{L}$ of calcium chloride. All CC cells were grown separately in Eagle's Minimum Essential Medium (EMEM, Gibco) with 10\% fetal bovine serum (FBS) (Life Technologies, Grand Island, NY, USA) supplementation, followed by incubation at $37^{\circ} \mathrm{C}$ in a tissue culture chamber with $95 \% \mathrm{O}_{2}$ and $5 \% \mathrm{CO}_{2}$.

\section{CC tissues}

Forty pairs of cervical carcinoma were supplied by the Affiliated Hospital of North Sichuan Medical College from November 2018 to January 2020. Study approval was granted by the Research Ethics Committee of Sichuan Academy of Medical Sciences \& Sichuan Provincial People's Hospital. The CC patients in this study were sampled before radiotherapy or chemotherapy, and clinical followup data were available for analysis. The use of tissues in this study followed the ethical guidelines of the Declaration of Helsinki (as revised in 2013), and each patient provided written informed consent for their tissues to be used in the scientific research.

\section{Cell transfection}

CC CasKi cells were obtained from the American Type Culture Collection (ATCC, Manassas, VA, USA). CasKi cells were cultivated in Eagle's Minimum Essential Medium (EMEM, Gibco) at $37{ }^{\circ} \mathrm{C}$ in a tissue culture chamber with $95 \% \mathrm{O}_{2}$ and $5 \% \mathrm{CO}_{2}$. miR-326 mimics for overexpression of the miR-326 level and control mimics (NC mimics), TCF4 for pcDNA3.0 (pc)-TCF4and pcDNA vector were also acquired. miR-326 mimics and pc-TCF4 were sourced from GenePharma (Shanghai, China). Lipofectamine
2000 reagent (Invitrogen; Thermo Fisher Scientific, Inc., Waltham, MA, USA) was used for transfection according to the protocol of the manufacturer.

\section{Quantitative real-time polymerase chain reaction (qRT-PCR)}

The TRIzol reagent kit (Invitrogen, Beijing, China) was employed for isolation of total RNA from CasKi cells, while reverse transcription was conducted using the PrimeScript RT Reagent Kit (Takara Biomedical Technology, Dalian, China); all procedures were carried out in line with the kits' instructions. The 2 SYBR Premix Ex Taq ${ }^{\mathrm{TM}}$ II (Takara, Takara Biomedical Technology, Dalian, China) was employed for qRT-PCR reaction system assembly, which was performed using the Bio-Rad CFX-96 (BioRad Laboratories, Hercules, CA, USA). Glyceraldehyde 3-phosphate dehydrogenase (GAPDH) was used for normalization. The relative expression levels of messenger RNA (mRNA) were calculated from the qRT-PCR data by applying the $2^{-\Delta \Delta C t}$ method. Primer sequences used in this study were as follows: U6-F: CTCGCTTCGGCAGCACA; U6-R: AACGCTTCACGAATTTGCGT; GAPDH-F: ACACCCACTCCTCCACCTTT; GAPDH-R: TTACTCCTTGGAGGCCATGT; miR-326-F: CCUCUGGGCCCUUCCUCCAG; miR-326-R: GCTGTCAACGATACGCTACCTA; TCF4-F : C C T GGCTAT GCA GGAATGTT; TCF 4-R : CAGGAGGCGTACAGGAAGAG. DNAStar 6.0 and Primer 5.6 software were employed for the primer design of miR-326 and TCF4 based on the sequence in the GenBank database.

\section{Western blotting}

Following the protocols of the manufacturer, RIPA lysis buffer (Beyotime Institute of Biotechnology, Shanghai, China) was used for cellular protein extraction. The BCA Protein Assay Kit (Beyotime Institute of Biotechnology, Shanghai, China) was selected for the detection of protein concentrations. The primary antibodies included rabbit antiKi67 antibody (1:25, ab833, Abcam), anti-proliferating cell nuclear antigen (PCNA) (1:1,000, \#13110, Cell Signaling), anti-Survivin (1:1,000, \#2808, Cell Signaling), anti-Bax (1:1,000, \#5023, Cell Signaling), anti-Bcl-2 antibody(1:500, ab196495, Abcam), anti-CD44 (1:100, \#37259, Cell Signaling), anti-SOX2 (1:1,000, \#14962, Cell Signaling), anti-OCT4 (1:1,000, \#2890, Cell Signaling), anti-ALDH1 
(1:1,000, \#54135, Cell Signaling), $\beta$-catenin (1:1,000, \#8480, Cell Signaling), c-Myc (1:1,000, \#18583, Cell Signaling), and cyclin D1 (1:1,000, \#2890, Cell Signaling). Goat anti-rabbit immunoglobin $\mathrm{G}$ ( $\mathrm{IgG}$ ) horseradish peroxidase (HRP)-conjugated secondary antibodies were incubated for $1 \mathrm{~h}$ at $37^{\circ} \mathrm{C}$. An automatic digital gel image analysis system, Bio-Rad CFX-96 (Bio-Rad, Hercules, CA, USA) was used to determine and analyze band density.

\section{Dual luciferase reporter assay}

Transfection of CasKi cells with miR-326 mimics was carried out, followed by co-transfection with TCF4-wild type (wt) or TCF4-mutant (mut) with Lipofectamine 2000 (Invitrogen; Thermo Fisher Scientific, Inc., Waltham, MA, USA). Luciferase assay analysis was conducted using a dualluciferase reporter assay system (Promega Corporation, Madison, WI, USA). After $48 \mathrm{~h}$, the cells were collected and subjected to examination for luciferase activity as previously described (25).

\section{Cell counting kit-8 (CCK-8) assay}

The effect of transfection with miR-326 or TCF4 or cotransfection with Sh-HULC and miR-377-5p inhibitor on the cell viability of CasKi cells was determined by CCK8 assay (Beyotime, China). HepG2 cells were respectively seeded into 96 -well plates at a density of $5 \times 10^{3}$ cells/well, with every experiment being repeated five times. Following incubation at $37^{\circ} \mathrm{C}$ for $24 \mathrm{~h}$, the addition of $20 \mu \mathrm{L}$ CCK8 reagent was made to each well, and the cells were further incubated for $1 \mathrm{~h}$ at $37^{\circ} \mathrm{C}$. Finally, a microplate reader (Thermo Fisher Scientific, USA) was used to read the optical density (OD) value at $450 \mathrm{~nm}$.

\section{Flow cytometry analysis}

CasKi cells with $90 \%$ confluency were twice subjected to rinsing with phosphate-buffered saline (PBS) buffer with trypsin added to the culture, and then placed in a $37^{\circ} \mathrm{C}$ incubator for $3 \mathrm{~min}$. After the cell synapses had disappeared, endothelial cell growth medium (EGM)-2 (Beijing Biterbo Biotechnology Co., Ltd., Beijing, China) containing 10\% FBS was added to terminate the digestion. The medium was aspirated with a pipette, and the bottom of the culture dish was repeatedly blown on to promote cell shedding. Transfer of the cell suspension to a $15-\mathrm{mL}$ centrifuge tube was followed by centrifugation for $5 \mathrm{~min}$. Then, the tissue and cells were resuspended by adding PBS buffer. An annexin V-FITC/propidium iodide (PI) apoptosis detection kit (Multisciences, Shanghai, China) was selected for detection of the apoptotic rate. Cells were washed three times with ice-cold PBS before resuspension and incubation with 5 $\mu \mathrm{L}$ of Annexin V-FIFC and $10 \mu \mathrm{L}$ of PI. A FACSAria Cell Sorter (BD Biosciences, Franklin Lakes, NJ, USA) was used for sample analysis. Data analyses were performed in Flow Jo (Tree Star, Ashland, OR, USA).

\section{Immunobistochemistry}

Sections of formalin-fixed, paraffin-embedded tissue were subjected to xylene deparaffinization and rehydration with gradient concentrations of ethanol. Then, overnight incubation of the tissue sections was conducted in the presence of rabbit anti-Ki67 antibody (1:25, ab833, Abcam), anti-PCNA (1:4,000, \#13110, Cell Signaling), anti-CD44 (1:200, \#37259, Cell Signaling), and anti-SOX2 (1:300, \#14962, Cell Signaling). The corresponding goat anti-rabbit IgG HRP-conjugated secondary antibodies were incubated at room temperature for $1 \mathrm{~h}$. The images were observed with an Olympus DX51 fluorescence microscope (Olympus, Tokyo, Japan). Data analysis was performed using image6.0 software.

\section{Xenograft tumor model assay}

Any experiment involving animals was conducted in line with the National Institutes of Health (NIH)Guide for the Care and Use of Laboratory Animals. The experiment underwent review by the Ethics Committee of Experimental Animal Management and Animal Welfare of Affiliated Hospital of North Sichuan Medical College, and subsequently received approval (no. 20181023c0600130[368]). To investigate the effects of miR-326 on tumor growth, an injection of $5.0 \times 10^{6}$ of CasKi cells was administered via the right flank near the hind leg of nude mice (6-week-old, female, athymic, BALB/ c nude mice). The mice were divided at random into two groups $(n=5)$ : the transfection control group and the miR326 mimic group.

\section{Statistical analyses}

All data are expressed as mean \pm SD. The two groups were compared by performing Student's $t$-test. The statistical data in the groups were analyzed by Duncan's test. Statistical analyses were conducted using SPSS software (version 19). 
A $\mathrm{P}$ value $<0.05$ was considered statistically significant.

\section{Results}

\section{MiR-326 regulated TCF4 expression by interacting with} 3'-UTR of TCF4

Previous research showed that the levels of miR-326 were significantly decreased in CC tissues and cell lines, which suppressed tumor cell proliferation, migration, and invasion $(13,26)$. The mRNA expression levels of miR-326 and TCF4 in human tissues and cells line were examined (normal cervical cell line Etc1/E6E7 and CC cell lines HeLa, C41, SiHa, and CaSki) using qRT-PCR. Results suggested that, compared with the control group, miR-326 was downregulated and TCF4 was upregulated in the tumor tissue group (Figure $1 A, B)$. In an interesting observation, a potential binding site with high conservation was found in the 3'-UTR of TCF4 mRNA (Figure 1C). To clarify the molecular mechanism underpinning the suppression of the proliferation and CSC-like property of CC cells by miR326 , the potential targets of miR-326 were searched for by using bioinformatics algorithms. miR-326 was found to contain complementary elements to the 3 '-UTR of the TCF4 regions (Figure 1C). The result predicted TCF4 as a potential target of miR-326.

pcDNA-TCF4 and miR-326 mimic transfection was performed in CasKi cells, respectively, resulting in a significant increase in the mRNA levels of TCF4 and miR326 (Figure 1D,E). A dual-luciferase reporter assay of the CasKi cells was conducted, and significant inhibition of luciferase activity was observed in CaSki cells cotransfected with the wt TCF4 3'-UTR and miR-326 (Figure 1F). Meanwhile, compared to the control group, there was a decrease in the protein expression levels of TCF4 in the miR-326 group, while the levels of TCF4 were comparatively increased in the TCF4 group (Figure 1G). Notably, the levels of TCF4 showed low contrast with the TCF4 group when miR-326 and TCF4 were cotransfected with CasKi cells. These results suggest that negative regulation of TCF4 expression was achieved through miR326's interaction with the 3'-UTR of TCF4 mRNA.

\section{MiR-326 inbibited the CasKi cell proliferation in vitro}

CCK-8 assay was used to further confirm whether miR-326 could inhibit CasKi cell proliferation via targeting TCF4. It was found that the upregulated expression of TCF4 promoted Caski cell viability compared with the control group (Figure $2 A$ ). Interestingly, CasKi cell viability was inhibited in the miR-326 + TCF4 group compared with the TCF4 group. In comparison with the controls, significant increases in the protein expression levels of ki67, PCNA, Survivin, and Bax occurred in the TCF4 group, whereas decreases in these expression levels were observed in the miR-326 group (Figure 2B). Moreover, the levels of Bal2 were reduced in the TCF4 group but were elevated in the miR-326 group. In comparison with the TCF4 group, the levels of ki67, PCNA, Survivin, and Bax were markedly downregulated in the miR-326 + TCF4 group, while the level of Bal-2 was upregulated. These observations suggested that miR-326 was able to exert an inhibitive effect on the proliferation ability of CasKi cells via targeting TCF4.

\section{MiR-326 suppressed the CSC-like property of CasKi cells in vitro}

CSCs are a major cause of poor clinical efficacy and increased mortality in cancer patients, and are involved in cell migration, invasion, metastasis, relapse, and treatment resistance. However, targeted killing of CSCs may be effective in eliminating tumor recurrence and metastasis. New evidence has suggested that miRNA is closely related to CSC (15) \{Huang, 2017 \#297\}\{Huang, 2017 \#297\} \{Huang, 2017 \#297\}. As further confirmation that miR326 can inhibit CSC-like properties in CasKi cells via targeting TCF4, it was found that, in comparison with the controls, the number of nodes and the sphere diameter were increased in the TCF4 group but reduced in the miR-203 group $(\mathrm{P}<0.05)$ (Figure $3 A, B, C)$. The number of nodes and diameter of spheres were markedly lower in the miR-326 + TCF4 group than in the TCF4 group. In comparison to the controls, the CSC markers of $\mathrm{CD} 133^{+}$were elevated in the TCF 4 group but suppressed in the miR-326 group. The CSC markers of $\mathrm{CD} 133^{+}$were markedly downregulated in the miR-326 + TCF4 group compared to the TCF4 group $(\mathrm{P}<0.05)$ (Figure 3D,E). Compared with the control group, the protein expression levels of CSC markers CD44, SOX2, OCT4, and aldehyde dehydrogenase 1 family member A1 (ALDH1) were elevated in the TCF 4 group but suppressed in the miR-326 group. The levels of the CSC markers CD44, SOX2, OCT4, and ALDH1 were markedly downregulated in the miR-326 + TCF4 group in contrast to the TCF4 group (Figure $3 F$ ). The results confirmed that miR-326 can inhibit the CSC-like property of CasKi cells 
A

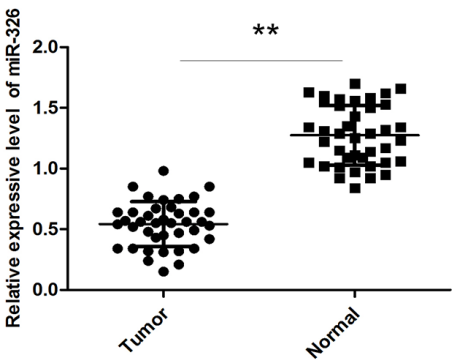

B

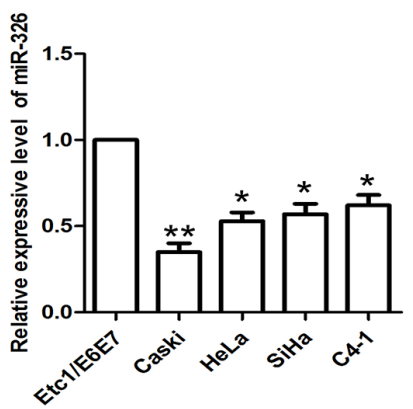

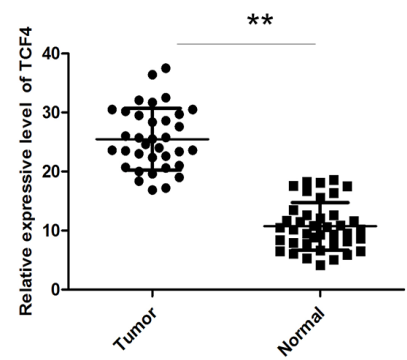

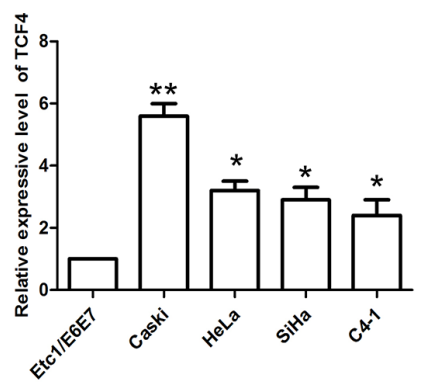

C

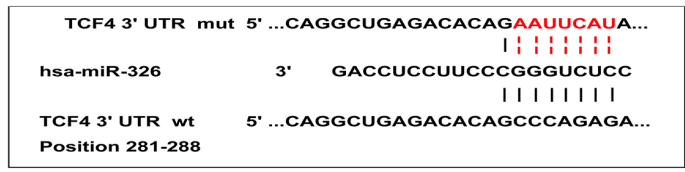

D

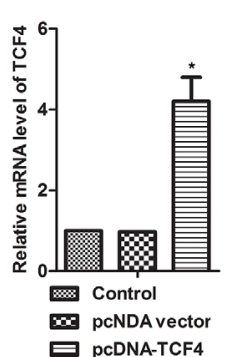

$\mathrm{E}$

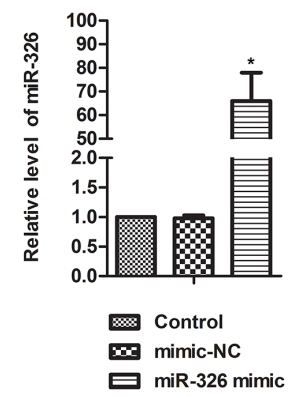

F
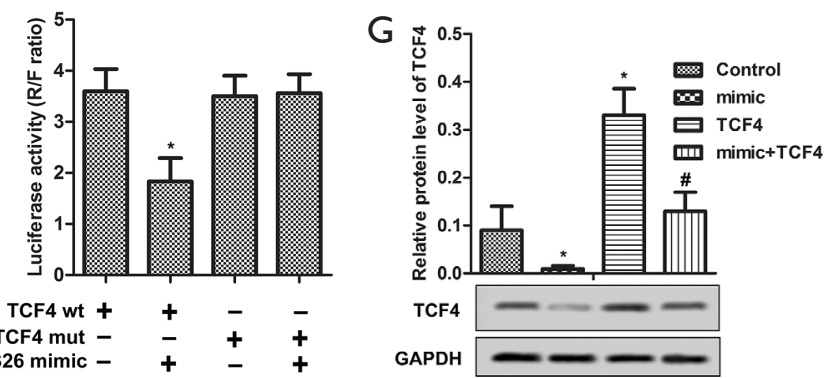

Figure 1 MiR-326 regulated TCF4 expression via its interaction with the 3'-UTR of TCF4. (A) The mRNA levels of miR-326 and TCF4 in a cervical cancer (CC) patient. (B) The mRNA levels of miR-326 and TCF4 in a normal cervical cell line, Etc1/E6E7, and CC cell lines HeLa, C4-1, SiHa, and CaSki by qRT-PCR. (C) Predicted binding sites between TCF4 and miR-326. Detection of the mRNA levels of TCF4 (D) and miR-326 (E) in CasKi cells was carried out by performing qRT-PCR. (F) Luciferase activity is presented relative to that of the control and mutant or wild-type TCF4 3'-UTR. (G) The relative protein levels of TCF4 were detected by western blot in the control group, the miR-326 group, the TCF4 group, and the miR-326 + TCF4 group $\left(^{*}, \mathrm{P}<0.05\right.$, compared with the control group; **, $\mathrm{P}<0.01$, compared with the control group; ${ }^{\prime}, \mathrm{P}<0.05$, compared with the TCF4 group). MiR-326, microRNA-326; TCF4, transcription factor 4; 3'UTR, three prime untranslated region; qRT-PCR, quantitative real-time polymerase chain reaction. 
A

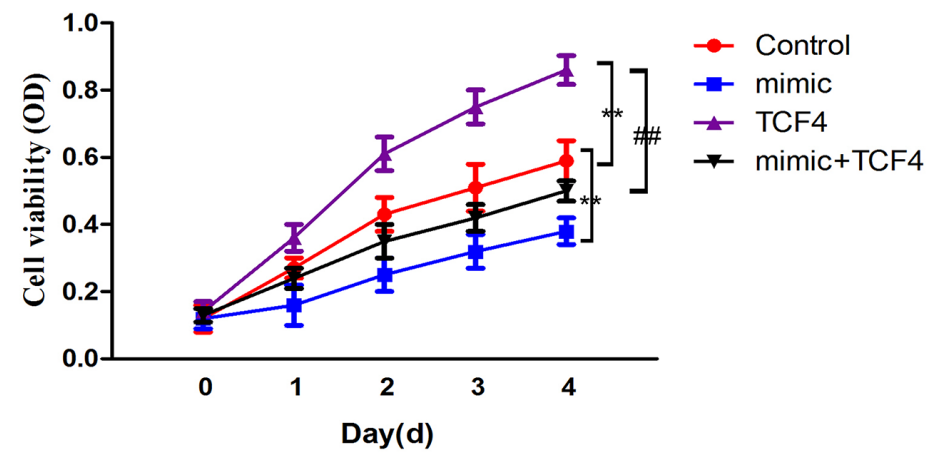

B
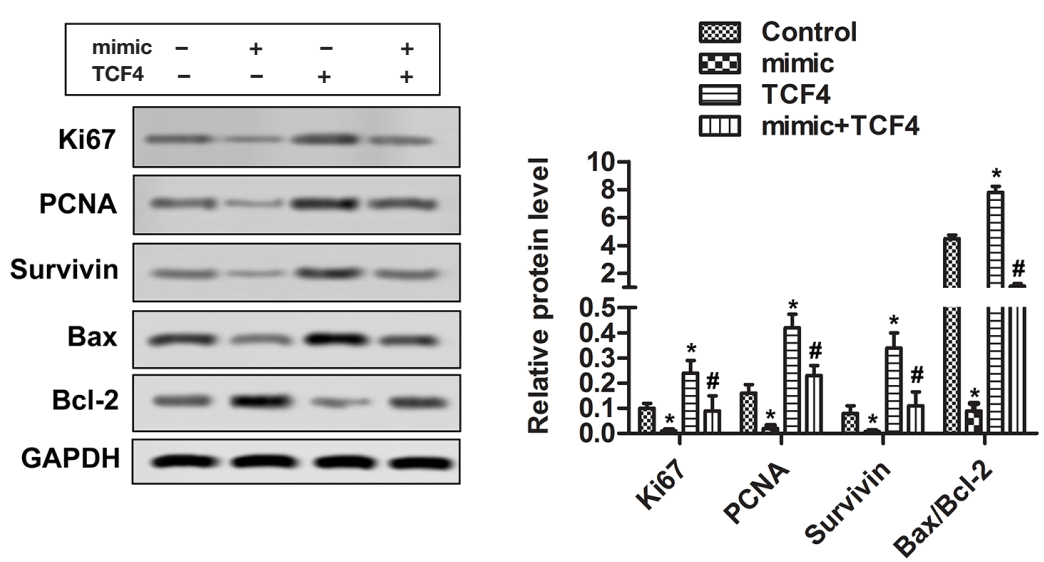

Figure 2 MiR-326 inhibited CasKi cell proliferation in vitro. CasKi cell viability was determined by CCK-8 assay (A). (B) Western blot was performed to detect the protein expressive levels of Ki-67, PCNA, Survivin, Bax, and Bal-2 (*, $\mathrm{P}<0.05$, compared with control group; ${ }^{*}$, $\mathrm{P}<0.05$, compared with the TCF4 group). MiR-326, microRNA-326; PCNA, proliferating cell nuclear antigen; TCF4, transcription factor 4.

via targeting TCF4.

\section{TCF4 adjusted the Wnt/B-catenin signaling}

The wingless-type (Wnt)/ $\beta$-catenin pathway is considered to be a major pathway with involvement in cervical carcinogenesis, which is a process comprising multiple steps. Therefore, its value as a potential biomarker or therapeutic target has been suggested (27). As TCF4 can adjust the $\mathrm{Wnt} / \beta$-catenin signaling pathway, the protein expression levels of $\beta$-catenin, c-Myc, and cyclin D1 were enhanced in the TCF4 group but reduced in the miR-203 group in comparison to the controls (Figure 4A). However, the levels of $\beta$-catenin, $c-M y c$, and cyclin D1 were downregulated in the miR-326 + TCF4 group compared to the TCF4 group. To further confirm if TCF4 can adjust Wnt/ $\beta$-catenin signaling pathway, DDK- 1 inhibitor was added. As shown in Figure 4B, the protein expression levels of TCF4, $\beta$-catenin, c-Myc, and cyclin D1 were lower in the DDK-1 group than in the control group. However, the levels TCF4, $\beta$-catenin, $\mathrm{c}-\mathrm{Myc}$ and cyclin D1 were upregulated in the DDK-1 + TCF4 group compared to the DDK-1 group (Figure 4B).

\section{MiR-326 inhibited tumor growth in vivo}

The xenograft tumor weight and volume was notably decreased in the miR-326 mice in comparison with the controls (Figure 5A,B), thus confirming miR-326's suppressive effect on tumor growth. In tumor tissues, the level of miR-326 expression in the miR-326 mice was elevated compared to that in the control group, while those of TCF4 were decreased (Figure 5C). The levels of cell proliferation (Ki-67 and PCNA) and CSC (CD44 and SOX2) markers were markedly downregulated in the miR-326 group compared with the control group (Figure $5 D, E, F$ ). $\beta$-catenin was inhibited in vivo (Figure $5 G$ ). These observations provided confirmation that miR-326 could exert an inhibitory effect on tumor growth in vivo. 
A

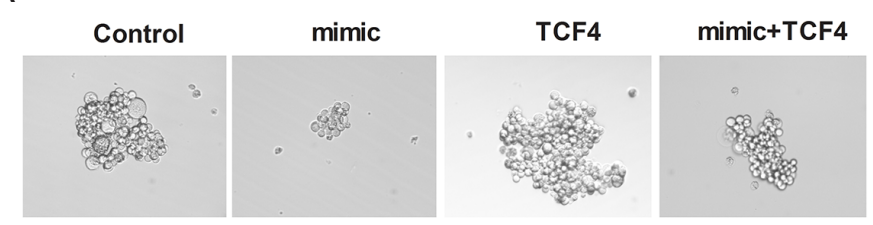

C

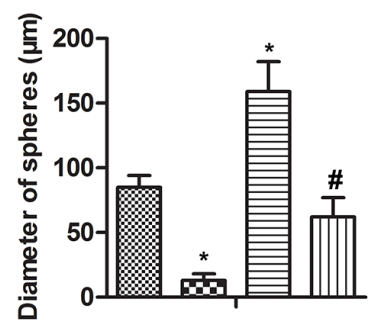

00 mimic

TCF4

ㅁI mimic+TCF4
B

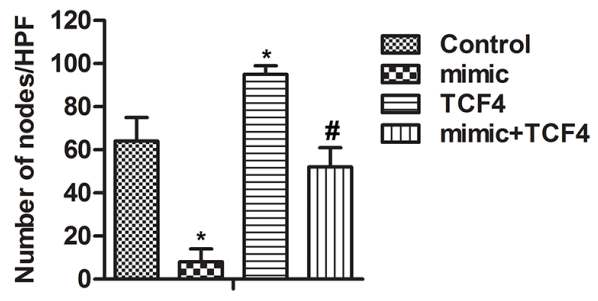

D

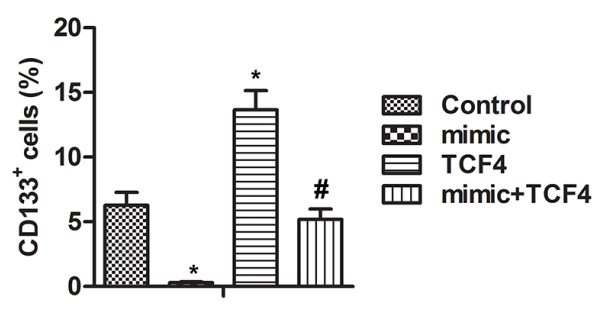

E
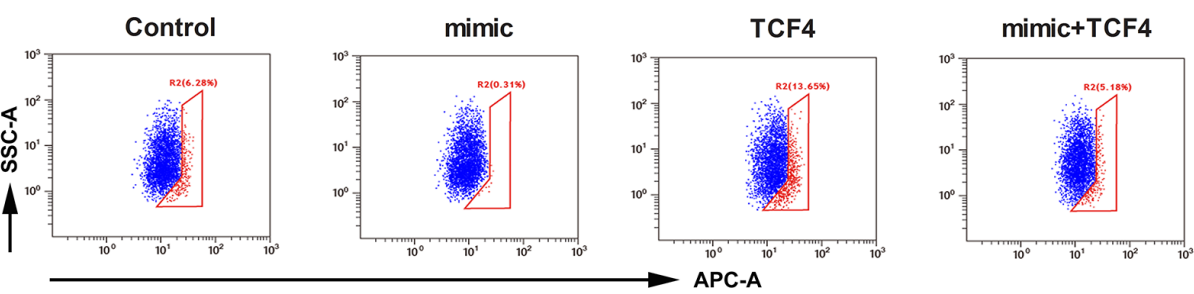

$\mathrm{F}$
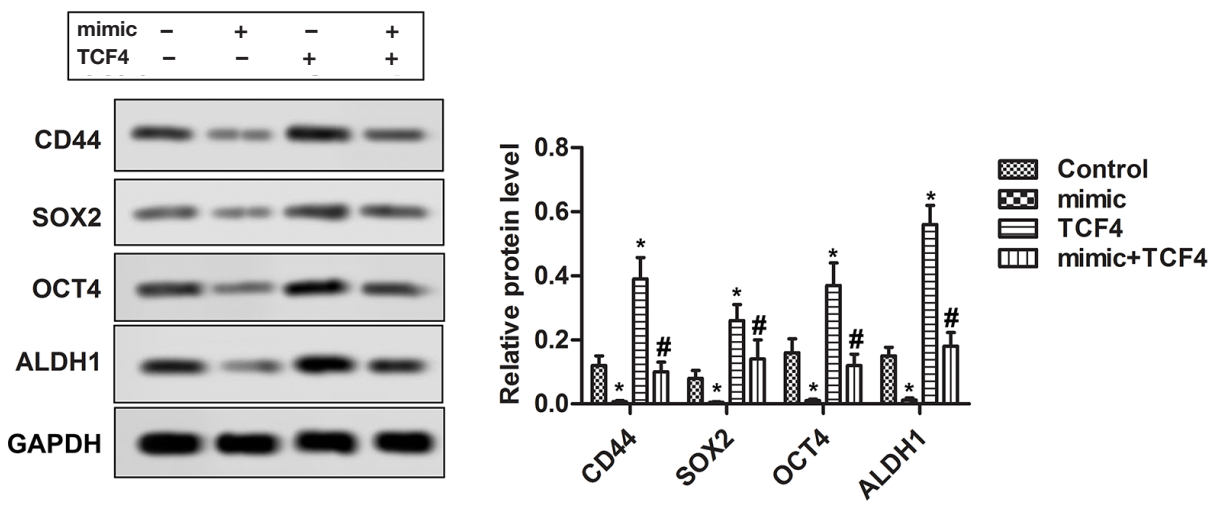

Figure 3 MiR-326 suppressed the CSC-like property of CasKi cells in vitro. (A) Tumor stem cell culture was carried out in a $37^{\circ} \mathrm{C}$ incubator containing $5 \% \mathrm{CO}_{2}$. Magnification 100x. (B) The number of nodes (C) and diameter of spheres (D) of tumor stem cells were measured with an electron microscope. Flow cytometry analysis of tumor stem cell markers of CD133+. (E) The proportion of tumor stem cells was calculated. (F) Western blot was performed to detect the protein expressive levels of the cancer stem cell markers CD44, SOX2, OCT4, and ALDH1 ( ${ }^{*}, \mathrm{P}<0.05$, compared with the control group; ${ }^{*}, \mathrm{P}<0.05$, compared with the TCF4 group).

\section{Discussion}

$\mathrm{CC}$ is among the most commonly diagnosed cancers in women and is a highly fatal and morbid disease in developing countries. Statistics show that China accounts for almost $25 \%$ of all CC cases globally (28). It has been found that in the early stages of CC, miRNA dysregulation promotes the disease by directly downregulating tumorassociated genes $(29,30)$. The relevant research has indicated that miRNA can act as an inhibitor of target gene function through degradation of mRNA or suppression of its translation in post-transcriptional regulation (31). The present research was designed to investigate the anticancer 

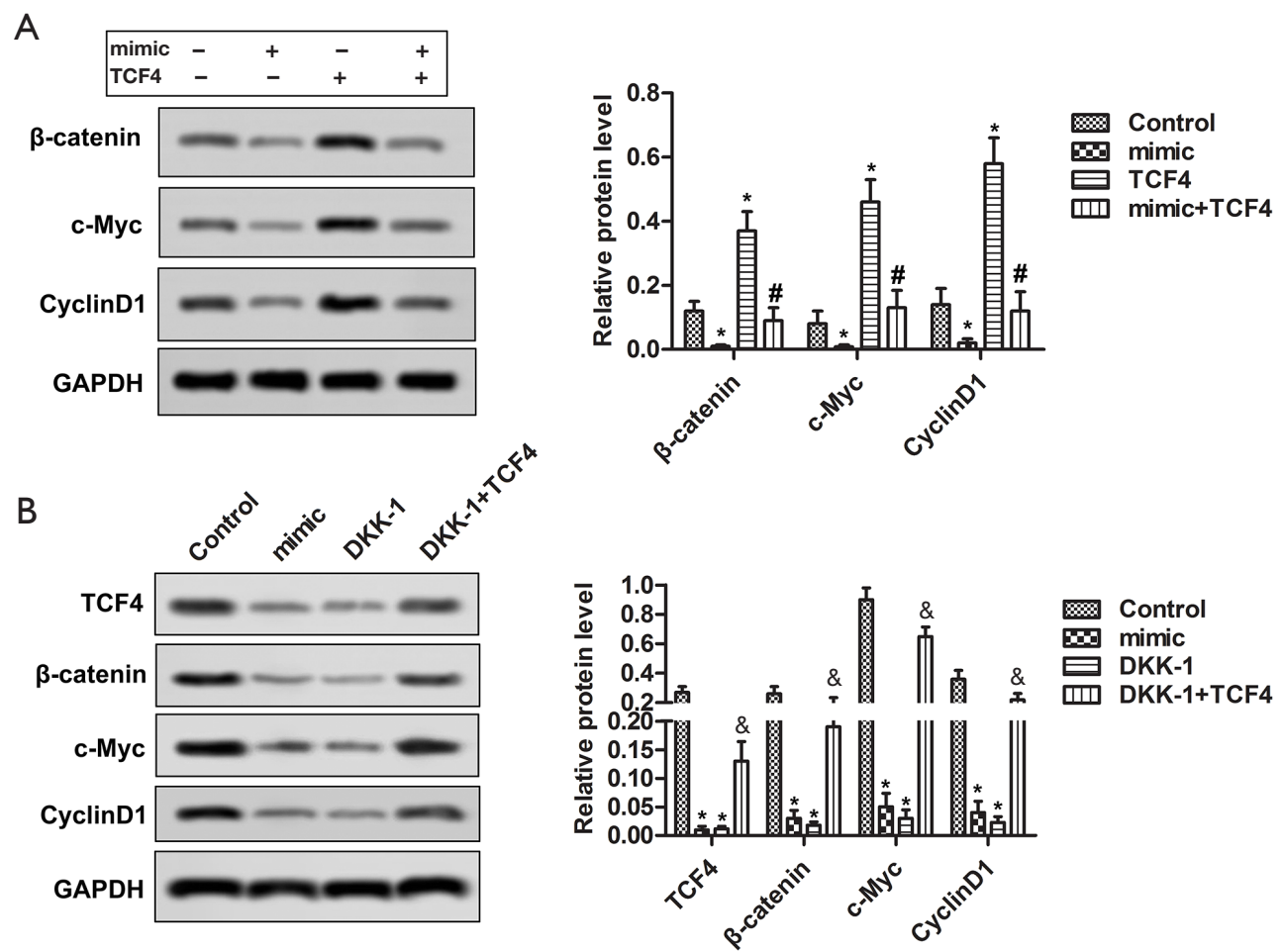

Figure 4 TCF4 adjusted the Wnt/ $\beta$-catenin signaling pathway. (A) The relative protein levels of $\beta$-catenin, c-Myc, and cyclin D1 were detected by western blot. The relative protein levels in each group were semiquantatively analyzed. (B) The relative protein levels of TCF4, $\beta$-catenin, c-Myc, and cyclin D1 were detected by western blot in the control group, the miR-203 group, the DDK-1 group, and the DDK1 plus TCF4 group. ${ }^{*}, \mathrm{P}<0.05$, compared with control group; ${ }^{*}, \mathrm{P}<0.05$, compared with the TCF4 group; ${ }^{*}, \mathrm{P}<0.05$, compared with the DDK-1 group).

function of miR-326 and the procancer function of TCF4 in CasKi cells. Importantly, the study indicated that protein expression levels of TCF4 were negatively regulated by miR-326, which interacted with the 3'-UTR of TCF4 mRNA. These results suggest that miR-326 can inhibit cancer cells by targeting TCF4.

Dysregulation of miR-326 is associated with various diseases. A previous study has indicated miR-326 is downregulated in lung cancer. When overexpressed, miR326 suppressed cell proliferation, migration, cell cycle, and apoptosis in both A549 and H838 cells, and it was found to inhibit tumor growth in nude mice $(32,33)$. Other research has shown that miR-326 markedly inhibits cell proliferation, colony formation, migration, and invasion, induces G0/G1 cell cycle arrest, and promotes apoptosis in prostatic carcinoma cells in vitro (34). Importantly, vascular endothelial growth factor C (VEGF-C) reduces miR326 expression but increases cortactin levels via c-Src signaling, thus enhancing CC invasiveness (35). Of further note, research has shown that miR-326 is expressed at markedly lower levels in CC tissue and in the HeLa, SiHa. and CaSki cell lines $(36,37)$. The overexpression of miR326 has been shown to decrease the proliferation, migration, and invasion abilities of CC cells (26). In the current study, CasKi cell proliferation in vitro and in vivo was suppressed by miR-326 when compared with the control group, which suggests that miR-326 can suppress the proliferation of CasKi cells in vitro.

Interaction of chromatin assembly factor 1 subunit A (CHAF1A) with TCF4 promotes gastric carcinogenesis through upregulation of c-MYC and CyclinD1 (CCND1) expression (38). A previous study demonstrated that miR-495 can inhibit the progression of non-small cell lung cancer by targeting TCF4 (39). Previous research has confirmed that the inhibitory effects of miR-591 expression on breast cancer cell proliferation and invasion are mediated by TCF4 (40). Crucially, it has been found that the overexpression of miR-299-3p can inhibit CC cell growth and invasion. Furthermore, TCF4 has been verified as a direct target of miR-299-3p, while the overexpression of TCF4 has been 
A

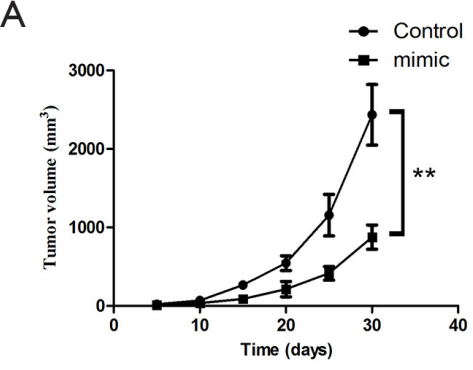

D

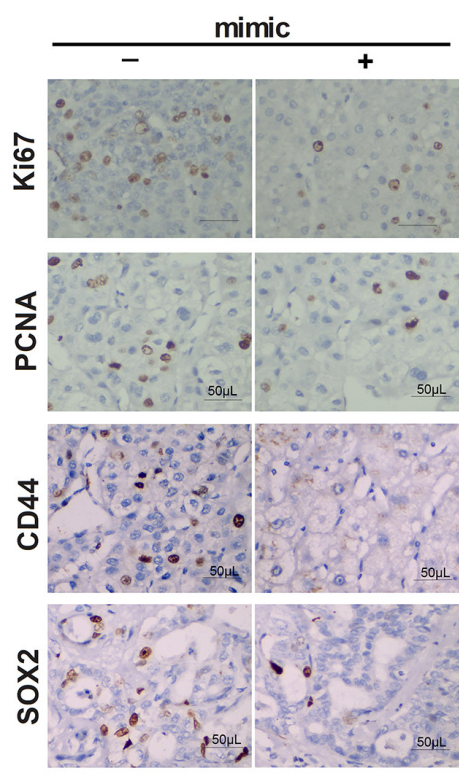

B

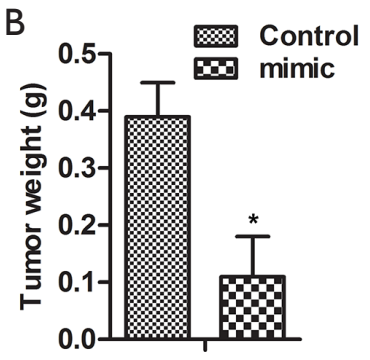

$\mathrm{E}$
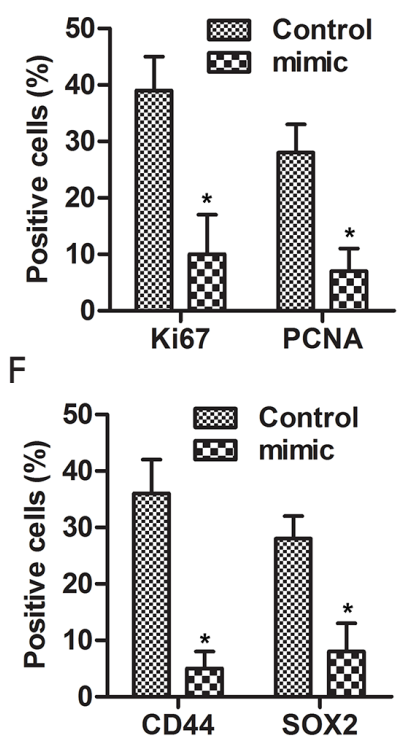
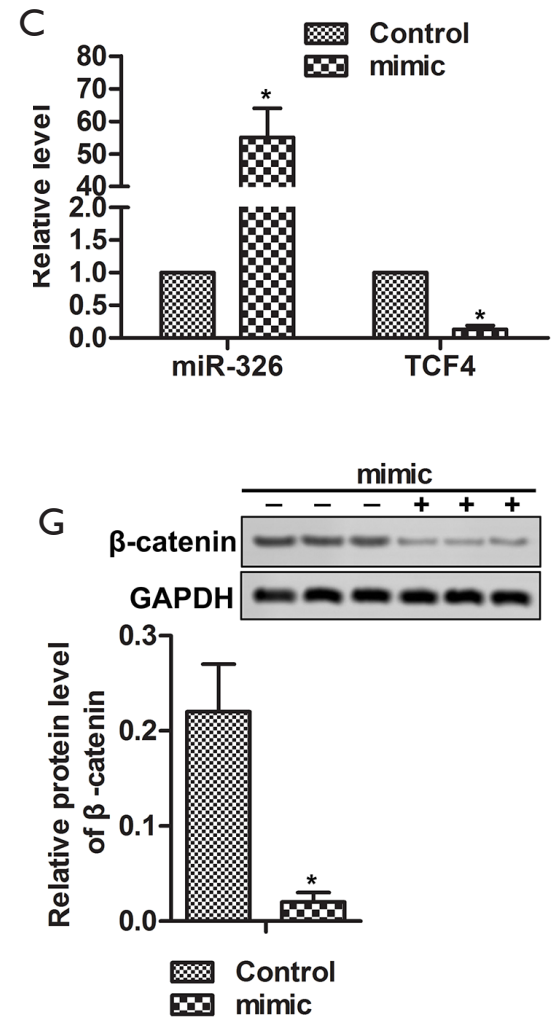

Figure 5 MiR-326 inhibited tumor growth in vivo. (A) The tumor volume was measured. (B) The histogram showed tumor weight (g). (C) The mRNA expression levels of miR-326 and TCF4 were detected by qRT-PCR. (D) Immunohistochemistry analysis of expression of (E) Ki-67, PCNA, and (F) CD44, SOX2. Magnification, $\times 400$. (G) Western blot analysis of $\beta$-catenin levels ${ }^{*}, \mathrm{P}<0.05$, compared with the control group).

proven to reverse the inhibitory effects of miR-299-3p on CC cell behaviors (41). Surprisingly, the present study found that CasKi cell proliferation in vitro was suppressed in the miR-326 + TCF4 group compared with the TCF4 group. This suggests that miR-326 can suppress the proliferation of CasKi cells in vitro via targeting TCF4.

CD133+ is a phenotypic marker of CSCs in CC (42). Herein, in comparison with the controls, the CSC markers of $\mathrm{CD}_{133^{+}}$in the TCF4 group were elevated but were reduced in the miR-203 group. Importantly, the CSC markers of $\mathrm{CD}_{133^{+}}$were markedly downregulated in the miR-326 + TCF4 group compared to the TCF4 group. Tyagi et al. (43) clarified the role of the HPV E6 oncogene in the maintenance of the stemness characteristics (stemness markers OCT4, SOX2, Nanog, Lrig1, and CD133) of CSCs in CC. Significant OCT4 marker upregulation has also been found in CC, which has an association with disease susceptibility and severity (44). Other research has indicated that ALDH1 and POU class 5 homeobox 1 (OCT4) may be utilized as biomarkers for the early detection of CC or monitoring the treatment of patients (45). In our study, the levels of the CSC markers CD44, SOX2, OCT4, and ALDH1 were markedly downregulated in the miR326 + TCF4 compared to the TCF4 group. Importantly, previous research has revealed that miR-326 can inhibit G-protein coupled receptor91(GPR91)/signal transducer and activator of transcription 3 (STAT3)/VEGF signaling pathway activation and markedly attenuate stem cell activity in endometrial carcinoma, both in vitro and in vivo (21). Collectively, these findings indicate that miR-326 can inhibit the CSC-like property of CasKi cell though targeting TCF4.

The Wnt signaling pathway is a key regulator of 
multiple biological processes, such as cell differentiation in embryogenesis, cell proliferation, cell migration, cell cycle, cell polarity, organogenesis during embryonic development, and tumor progression (46). To date, a considerable amount of research has evidenced $\mathrm{Wnt} / \beta$-catenin signaling as being a contributor to the deterioration of CC (47). TCF4, in conjunction with $\beta$-catenin coactivator, functions as the main transcriptional mediator of the canonical Wnt signaling pathway in the intestinal epithelium (48). The CasKi cell line is a metastatic cell line originating from the small intestine. Human CC CasKi cells have been shown to exhibit markedly increased levels of $\beta$-catenin and Twist, which can be treated with the anti-cancer agent lithium chloride (49). In the present study, cyclin D1, $\beta$-catenin, and c-Myc were inhibited in the miR-326 group in contrast to the control group, but appeared to be elevated in the TCF4 group, while cyclin D1, $\beta$-catenin, and $\mathrm{c}-\mathrm{Myc}$ were decreased in the miR-326 + TCF4 group in comparison with the TCF4 group. However, when DDK-1 inhibitor was added in vitro, cyclin D1, $\beta$-catenin, and c-Myc were increased in the DDK-1 plus TCF4 group compared to the DDK-1 group, which was in agreement with the in vivo results. Overall, these findings suggest that TCF4 can activate the Wnt signaling pathway, while miR-326 can inhibit the Wnt signaling pathway.

Xenograft tumor weight and volume were notably lower in the miR-326 group than in the control group. In tumor tissues, the cell proliferation (Ki67 and PCNA) and CSC (CD44 and SOX2) marker levels were downregulated markedly in vivo in comparison to the non-transfection miR-326 group, while $\beta$-catenin was inhibited in vivo. These observations provided verification that miR-326 had an inhibitory effect on tumor growth in vivo.

In conclusion, using bioinformatics analysis, we were able to confirm TCF4 as a potential target gene of miR326. Overexpression of miR-326 markedly reduced TCF4 expression at the protein level. miR-326 suppressed the proliferation and CSC-like property of CasKi cells in vitro via targeting TCF4. Furthermore, miR-326 could inhibit the $W n t / \beta$-catenin pathway, while TCF4 could activate the $\mathrm{Wnt} / \beta$-catenin pathway. Importantly, miR-326 inhibited tumor growth in vivo. These findings are promising as they offer a new target or the treatment of $\mathrm{CC}$, but further data collection is needed to validate the impact on CC.

\section{Acknowledgments}

Funding: This study was supported by the Affiliated Hospital of North Sichuan Medical College.

\section{Footnote}

Reporting Checklist: The authors have completed the ARRIVE reporting checklist. Available at http://dx.doi. org/10.21037/atm-20-6830

Data Sharing Statement: Available at http://dx.doi. org/10.21037/atm-20-6830

Conflicts of Interest: All authors have completed the ICMJE uniform disclosure form (available at http://dx.doi. org/10.21037/atm-20-6830). The authors have no conflicts of interest to declare.

Ethical Statement: The authors are accountable for all aspects of the work in ensuring that questions related to the accuracy or integrity of any part of the work are appropriately investigated and resolved. The study was approved by the Research Ethics Committee of Sichuan Academy of Medical Sciences \& Sichuan Provincial People's Hospital. All tissues were used according to ethical guidelines of the Declaration of Helsinki (as revised in 2013), and the written informed consent for scientific research was obtained from all patients. All animal experiments were carried out in accordance with the National Institutes of Health (NIH) Guide for the Care and Use of Laboratory Animals. The Ethics Committee of Experimental Animal Management and Animal Welfare of Affiliated Hospital of North Sichuan Medical College also reviewed and approved the experiment (no. 20181023c0600130[368]).

Open Access Statement: This is an Open Access article distributed in accordance with the Creative Commons Attribution-NonCommercial-NoDerivs 4.0 International License (CC BY-NC-ND 4.0), which permits the noncommercial replication and distribution of the article with the strict proviso that no changes or edits are made and the original work is properly cited (including links to both the formal publication through the relevant DOI and the license). See: https://creativecommons.org/licenses/by-nc-nd/4.0/.

\section{References}

1. Tsikouras P, Zervoudis S, Manav B, et al. Cervical cancer: screening, diagnosis and staging. J BUON 2016;21:320-5.

2. Siegel R, Naishadham D, Jemal A. Cancer statistics, 2012. CA Cancer J Clin 2012;62:10-29. 
3. Zhang W, He W, Shi Y, et al. High Expression of KIF20A Is Associated with Poor Overall Survival and Tumor Progression in Early-Stage Cervical Squamous Cell Carcinoma. PLoS One 2016;11:e0167449.

4. Babion I, Jaspers A, van Splunter AP, et al. miR-95 p Exerts a Dual Role in Cervical Cancer and Targets Transcription Factor TWIST1. Cells 2019;9:65.

5. Chen J, Ao L, Yang J. Long non-coding RNAs in diseases related to inflammation and immunity. Ann Transl Med 2019;7:494.

6. Almeida MI, Reis RM, Calin GA. MicroRNA history: discovery, recent applications, and next frontiers. Mutat Res 2011;717:1-8.

7. He Y, Lin J, Ding Y, et al. A systematic study on dysregulated microRNAs in cervical cancer development. Int J Cancer 2016;138:1312-27.

8. Pan S, Liu Y, Liu Q, et al. Corrigendum to "HOTAIR/ miR-326/FUT6 axis facilitates colorectal cancer progression through regulating fucosylation of CD44 via PI3K/AKT/mTOR pathway" [BBA - Mol. Cell Res. 1866 (2019) 750-760]. Biochim Biophys Acta Mol Cell Res 2019;1866:1353.

9. Mo Y, He L, Lai Z, et al. Gold nano-particles (AuNPs) carrying miR-326 targets PDK1/AKT/c-myc axis in hepatocellular carcinoma. Artif Cells Nanomed Biotechnol 2019;47:2830-7.

10. Ghaemi Z, Soltani BM, Mowla SJ. MicroRNA-326 Functions as a Tumor Suppressor in Breast Cancer by Targeting ErbB/PI3K Signaling Pathway. Front Oncol 2019;9:653.

11. Liu W, Zhang B, Xu N, et al. miR-326 regulates EMT and metastasis of endometrial cancer through targeting TWIST1. Eur Rev Med Pharmacol Sci 2017;21:3787-93.

12. Tang Q, Chen Z, Zhao L, et al. Circular RNA hsa_ circ_0000515 acts as a miR-326 sponge to promote cervical cancer progression through up-regulation of ELK1. Aging (Albany NY) 2019;11:9982-99.

13. Jiang $H$, Liang $M$, Jiang $Y$, et al. The lncRNA TDRG1 promotes cell proliferation, migration and invasion by targeting miR-326 to regulate MAPK1 expression in cervical cancer. Cancer Cell Int 2019;19:152.

14. Sareddy GR, Panigrahi M, Challa S, et al. Activation of Wnt/beta-catenin/Tcf signaling pathway in human astrocytomas. Neurochem Int 2009;55:307-17.

15. Liu L, Zeng Z, Yi J, et al. Expression and clinical significance of transcription factor 4 (TCF4) in epithelial ovarian cancer. Cancer Biomark 2019;24:213-21.

16. Ji X, Guo H, Yin S, et al. miR-139-5p functions as a tumor suppressor in cervical cancer by targeting TCF4 and inhibiting Wnt/beta-catenin signaling. Onco Targets Ther 2019;12:7739-48.

17. Chen G, Han N, Li G, et al. Time course analysis based on gene expression profile and identification of target molecules for colorectal cancer. Cancer Cell Int 2016;16:22.

18. Huang R, Rofstad EK. Cancer stem cells (CSCs), cervical CSCs and targeted therapies. Oncotarget 2017;8:35351-67.

19. Kidd EA, Grigsby PW. Intratumoral metabolic heterogeneity of cervical cancer. Clin Cancer Res 2008;14:5236-41.

20. Huang Z, Wu T, Liu AY, et al. Differentiation and transdifferentiation potentials of cancer stem cells. Oncotarget 2015;6:39550-63.

21. Gao Y, Qian H, Tang X, et al. Superparamagnetic iron oxide nanoparticle-mediated expression of miR-326 inhibits human endometrial carcinoma stem cell growth. Int J Nanomedicine 2019;14:2719-31.

22. Babashah S, Sadeghizadeh M, Hajifathali A, et al. Targeting of the signal transducer Smo links microRNA-326 to the oncogenic Hedgehog pathway in CD34+ CML stem/ progenitor cells. Int J Cancer 2013;133:579-89.

23. Huang R, Rofstad EK. Cancer stem cells (CSCs), cervical CSCs and targeted therapies. Oncotarget 2017;8:35351-67.

24. Rao QX, Yao TT, Zhang BZ, et al. Expression and functional role of ALDH1 in cervical carcinoma cells. Asian Pac J Cancer Prev 2012;13:1325-31.

25. Ji Q, Xu X, Xu Y, et al. miR-105/Runx2 axis mediates FGF2-induced ADAMTS expression in osteoarthritis cartilage. J Mol Med (Berl) 2016;94:681-94.

26. Zhang P, Kong F, Deng X, et al. MicroRNA-326 suppresses the proliferation, migration and invasion of cervical cancer cells by targeting ELK1. Oncol Lett 2017;13:2949-56.

27. Bahrami A, Hasanzadeh M, ShahidSales S, et al. Clinical Significance and Prognosis Value of Wnt Signaling Pathway in Cervical Cancer. J Cell Biochem 2017;118:3028-33.

28. Chen $\mathrm{W}$, Zheng R, Baade PD, et al. Cancer statistics in China, 2015. CA Cancer J Clin 2016;66:115-32.

29. Wang F, Li B, Xie X. The roles and clinical significance of microRNAs in cervical cancer. Histol Histopathol 2016;31:131-9.

30. Srivastava SK, Ahmad A, Zubair H, et al. MicroRNAs in gynecological cancers: Small molecules with big 
implications. Cancer Lett 2017;407:123-38.

31. Hauptman N, Glavac D. MicroRNAs and long noncoding RNAs: prospects in diagnostics and therapy of cancer. Radiol Oncol 2013;47:311-8.

32. Wang R, Chen XF, Shu YQ. Prediction of non-small cell lung cancer metastasis-associated microRNAs using bioinformatics. Am J Cancer Res 2015;5:32-51.

33. Wang R, Chen X, Xu T, et al. MiR-326 regulates cell proliferation and migration in lung cancer by targeting phox $2 \mathrm{a}$ and is regulated by HOTAIR. Am J Cancer Res 2016;6:173-86.

34. Liang X, Li Z, Men Q, et al. miR-326 functions as a tumor suppressor in human prostatic carcinoma by targeting Mucin1. Biomed Pharmacother 2018;108:574-83.

35. Cheng Y, Jiang S, Yuan J, et al. Vascular endothelial growth factor $\mathrm{C}$ promotes cervical cancer cell invasiveness via regulation of microRNA-326/cortactin expression. Gynecol Endocrinol 2018;34:853-8.

36. Zhang P, Kong F, Deng X, et al. MicroRNA-326 suppresses the proliferation, migration and invasion of cervical cancer cells by targeting ELK1. Oncol Lett 2017;13:2949-56.

37. Cao Y, Li J, Jia Y, et al. CircRNA circ_POLA2 Promotes Cervical Squamous Cell Carcinoma Progression via Regulating miR-326/GNB1. Front Oncol 2020;10:959.

38. Zheng L, Liang X, Li S, et al. CHAF1A interacts with TCF4 to promote gastric carcinogenesis via upregulation of c-MYC and CCND1 expression. EBioMedicine 2018;38:69-78.

39. Zheng HE, Wang G, Song J, et al. MicroRNA-495 inhibits the progression of non-small-cell lung cancer by targeting TCF4 and inactivating Wnt/beta-catenin pathway. Eur Rev Med Pharmacol Sci 2018;22:7750-9.

40. Huang X, Tang F, Weng Z, et al. MiR-591 functions as tumor suppressor in breast cancer by targeting TCF4 and

Cite this article as: Zhang J, He H, Wang K, Xie Y, Yang Z, Qie M, Liao Z, Zheng Z. miR-326 inhibits the cell proliferation and cancer stem cell-like property of cervical cancer in vitro and oncogenesis in vivo via targeting TCF4. Ann Transl Med 2020;8(24):1638. doi: 10.21037/atm-20-6830 inhibits Hippo-YAP/TAZ signaling pathway. Cancer Cell Int 2019;19:108.

41. Yu Y, Zhao JD, Yang H. MiR-299-3p inhibits proliferation and invasion of cervical cancer cell via targeting TCF4. Eur Rev Med Pharmacol Sci 2019;23:5621-7.

42. Javed S, Sharma BK, Sood S, et al. Significance of CD133 positive cells in four novel HPV-16 positive cervical cancer-derived cell lines and biopsies of invasive cervical cancer. BMC Cancer 2018;18:357.

43. Tyagi A, Vishnoi K, Mahata S, et al. Cervical Cancer Stem Cells Selectively Overexpress HPV Oncoprotein E6 that Controls Stemness and Self-Renewal through Upregulation of HES1. Clin Cancer Res 2016;22:4170-84.

44. Tiwari D, Ray Das C, Sultana R, et al. Impact of modulation of telomerase and cancer stem-cell marker OCT4 axis in cervical cancer pathogenesis with underlying HPV16 infection. J Cell Biochem 2020;121:2782-91.

45. Tulake W, Yuemaier R, Sheng L, et al. Upregulation of stem cell markers ALDH1A1 and OCT4 as potential biomarkers for the early detection of cervical carcinoma. Oncol Lett 2018;16:5525-34.

46. MacDonald BT, Tamai K, He X. Wnt/beta-catenin signaling: components, mechanisms, and diseases. Dev Cell 2009;17:9-26.

47. Shinohara A, Yokoyama Y, Wan X, et al. Cytoplasmic/ nuclear expression without mutation of exon 3 of the beta-catenin gene is frequent in the development of the neoplasm of the uterine cervix. Gynecol Oncol 2001;82:450-5.

48. van Es JH, Haegebarth A, Kujala P, et al. A critical role for the Wnt effector Tcf4 in adult intestinal homeostatic selfrenewal. Mol Cell Biol 2012;32:1918-27.

49. Sun X, Liu Y. Activation of the Wnt/beta-catenin signaling pathway may contribute to cervical cancer pathogenesis via upregulation of Twist. Oncol Lett 2017;14:4841-4. 